\title{
QUALITATIVE ANALYSIS OF RESEARCH PROTOCOLS PRESENTED TO THE INSTITUTIONAL ETHICS COMMITTEE IN A TEACHING INSTITUTION: A RE- SEARCH ON RESEARCH
}

\author{
Dr. Divya John Stephy*, Dr. C. Ramachandra Bhat, Dr. T. Aruna, Dr. J. Komathi \\ Government Kilpauk Medical College, Chennai, Tamilnadu- 600010, India \\ *Corresponding Author's Email: div_stephy@yahoo.com
}

Received 20 Jan 2015; Review Completed 19 Feb 2015; Accepted 14 March 2015, Available online 15 March 2015

\begin{abstract}
:
Introduction: Increase in research aptitude among students has resulted in a rise in the protocol numbers that get submitted to the Institutional Ethics Committee (IEC) for the purpose of recognition in dissertation, publications and forum presentations. Approval of research protocols by the IEC is considered the first barrier for most student researchers. Lack of solid concepts regarding dynamics involved in research seems to have resulted in unwarranted fear among students to pursue research. The current analysis attempts to identify areas of lacunae by retrospective reflection on the protocols submitted to the IEC in a teaching institution. Methodology: Retrospective Qualitative Analysis of 102 Protocols submitted by postgraduate medical students between January, 2013 to August, 2013 to the IEC were assessed by the following parameters: - Scientific and technical soundness of protocols, Ethical considerations and risk minimization strategies employed, Adequacy of documentation and scrutiny of Informed consent process, Temporal relationship between submission of protocols and commencement of the research procedures. Results: $79.41 \%$ of protocols were approved by the IEC. $95 \%$ of protocols were in proper format and adequate documentation. $90 \%$ of the protocols was submitted for approval prior to the commencement of study. $20.59 \%$ of the submitted protocols were denied approval on the first hearing by the IEC. Discussion: We infer that majority of the postgraduate students are aware about the existence of guidelines in clinical research. The most common errors leading to non approval of research protocols are due to misjudgement regarding technical feasibility in available infrastructure, non comprehensive review of literature, non-addressal of ethical guidelines/risk minimisation strategies towards participants and inadequate documentation.

Keywords: Biomedical Research, Institutional Ethics Committee, Qualitative Research
\end{abstract}

\section{INTRODUCTION:}

Research is an inborn trait present in all humans. Asking questions, searching for answers and expanding their knowledge databases have been crucial in the world of science. The field of Medicine is no exception to this trend as well.

Primitive men gathered the knowledge of healing and medicine by observing nature and by personal experiences after consuming certain herbs and berries as remedies for their illnesses. However, these blind "trial and error"- based experimentation were termed obsolete and crude by the pioneers of Clinical Research. ${ }^{1}$

Francois Magendie, a French scientist of the seventeenth century dictated, "Facts and Facts alone are the basis of science". ${ }^{2}$ The first documented clinical trial dates back to 1747, when James Lind established the importance of citrus fruits in Scurvy among sailors. This clinical study is a landmark in the field of Biomedical Research until date. ${ }^{3}$

In the quest to unravel the mysteries of medical science, an enthusiastic researcher often gets biased in his/her decision-making. The distinction between the right and wrong decisions in medical research is not always clear.
While ethics is the correct behaviour dictated by one's own moral integrity, it forms the skeletal framework of Biomedical Research, upholding the rights and dignity of the participants and at the same time providing maximum benefits and minimal harm to them. ${ }^{4}$

Clashes in opinion arise based on two normative theories of Ethics - Utilitarianism and Kantianism. Utilitarianism means to do greatest good for greatest number, while Kantianism means to always act right and do well for everyone. ${ }^{5}$

Built upon this ethical foundation, various key elements are erected to complete the infrastructure of Biomedical Research. These key elements include a solid scientific basis and rationale to the study hypothesis, a comprehensive study protocol, comprehensive and apriori statement of various research procedures and protection of participants' rights, risk- mitigation and minimization strategies, etc.

Increase in research aptitude among medical students has resulted in a profound increase in the quanta of student investigator- initiated research projects. Aspiring student researchers are often biased about their 
research. In their enthusiasm to solve their study question, certain of these key elements of Research may be compromised at times by them. Adding to the momentum, the recent amendment by The Medical Council of India states that research publication is mandatory for academic and promotional escalation. ${ }^{6}$

Institutional Ethics Committee (IEC) is an independent multidisciplinary and multi-sectorial body at the Institutional level that scrutinizes the scientific and ethical integrity protocols submitted by the student investigators. The IEC is the quality check for the soundness of scientific and technical content, an advisory to uplift the participants' rights and a bridge to strengthen the biomedical ethics in clinical research procedures. ${ }^{7}$

Non- approval of protocols submitted to IEC often results in disappointment and unwarranted fear towards research among medical students. Many times, aspiring researchers' first attempts to pursue research are met with resistance during IEC meetings. The current study is an attempt to identify areas of lacunae in the protocols submitted by the student researchers (postgraduate and undergraduate medical students) and to come up with suggestions to bridge the inadequacies in current protocols.

\section{MATERIALS AND METHODS: RESULTS:}

Among the 102 protocols analysed, 81 (79.41\%) were approved, $8(7.85 \%)$ were rejected and the rest
Analytical study on the protocols submitted to the IEC was done at Government Kilpauk Medical College (GKMC), Chennai, Tamilnadu. Study procedures were commenced after obtaining approval from IEC, (GKMC).Our IEC convenes every first Thursday of the month with a quorum of 12 members, constituted as per ICMR guidelines. All of the 102 protocols submitted by postgraduate medical students between January, 2013 to August, 2013 were included in the study. Data was collected from these protocols and Minutes of meeting records of the IEC. The protocols were analysed using for the following parameters:

1. Scientific and technical soundness of research question

2. Ethical considerations and risk minimization strategies employed

3. Adequacy of documentation and scrutiny of the Informed consent process

4. Format of the protocols

5. Temporal relationship between submission of protocols and commencement of the research procedures

The raw data was organized and charted on Microsoft excel sheets categorically.

Results were expressed with descriptive statistical methods.

$13(12.75 \%)$ were advised to be resubmitted with relevant modifications by the IEC

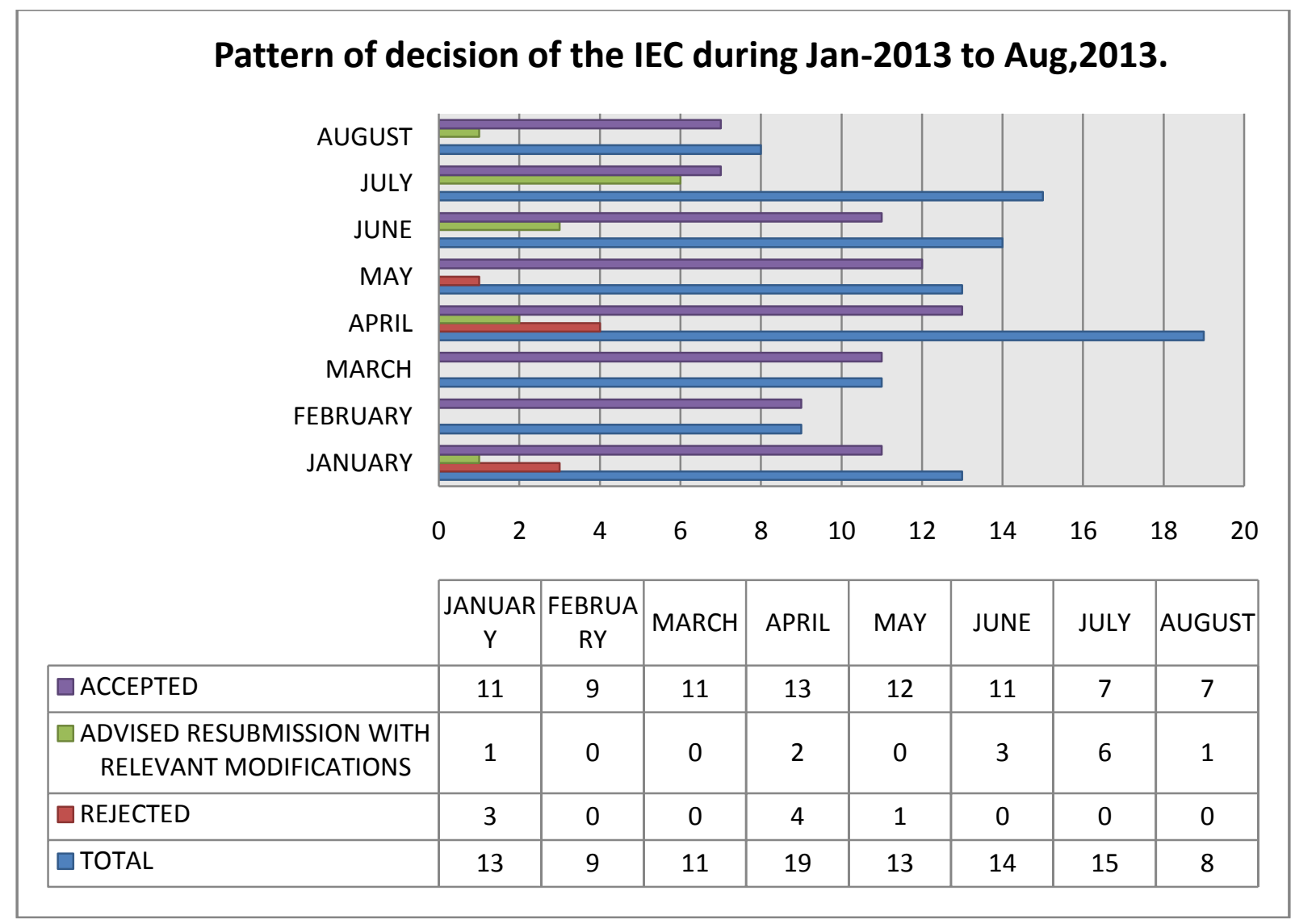


1. Scientific and Technical soundness of research question

Scientific and technical soundness of the submitted protocols were reviewed based on expert opinion reports in the IEC meeting records. The following deficits were noted in the protocols that were either rejected or required relevant modifications.

\section{ERRORS IN SCIENTIFIC AND TECHNICAL ASPECTS OF RESEARCH}

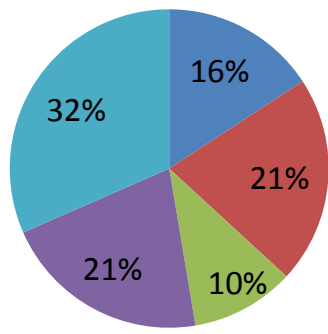

- INADEQUATE SAMPLE SIZE

- INADEQUATE DURATION OF STUDY

- IMPROPER METHODOLOGY

- LACK OF ADEQUATE LITERATURE REFERENCES

- LACK OF PERMISSION FROM CONCERNED DEPARTMENTS

2. Ethical considerations and risk minimization strategies employed :-

Four out of the eight rejected protocols were due to inadequate consideration of the ethical guidelines in biomedical research, incoherent and inadequate informed consent forms and poor statement of the risk-benefit analysis. Our IEC rejected research projects, when the Principal Investigator failed to produce coherent, transparent and comprehensible documents on Participant Information Sheet and Informed Consent.

3. Adequacy of documentation and scrutiny on Informed consent :-

Out of the six protocols that got rejected under this category, two protocols were rejected due to inadequate documentation, mostly due to lack of obtaining permission to conduct the study from the allied departments. Other four protocols were rejected in view of absence of templates of Informed consent and Patient Information/ Education documents in the vernacular language of the participants.

4. Format of scientific medical protocol :-

Each IEC has its own templates for ensuring standard and quality of the scientific medical protocol submitted by the student researchers. Our institution website provides a downloadable template of this format to guide student researchers Due to the fixity of this documentation format, all the protocols met the required standards.

5. Temporal relationship between submission of protocol and commencement of study

\section{SUBMISSION OF PROTOCOL IN RELATION TO CONDUCT OF STUDY}

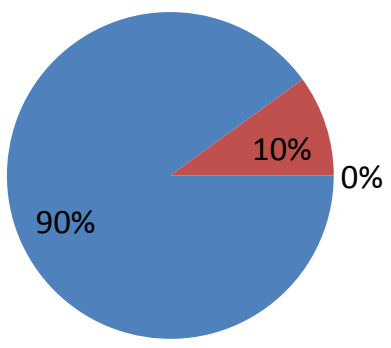

- PRIOR TO CONDUCT OF STUDY

- DURING THE PERIOD OF STUDY

AFTER COMPLETION OF STUDY

All the submitted protocols were submitted prior/ during the study period. Ninety percent of the protocols were submitted before the commencement of the study, rest $10 \%$ of the studies were presented to IEC during the study period.

\section{DISCUSSION:}

Increase in research aptitude, increasing avenues for research and recognition of research- oriented fields as career option have resulted in a unanimous and universal acceptance of Research among medical students. With increasing stringency in Research guidelines, a student researcher considers approval of their protocol as a Himalayan feat.

Based on the current study, the most common lacunae areas leading to non- approval of research protocols are due to inadequate consideration of the technical feasibility, poorly researched scientific background, incoherently stated informed consent/ Participant Information Sheet and risk minimisation strategies for participants' safety and inadequacy of necessary documents .

In a Questionnaire survey conducted by Jadhav M \& Bhatt $\mathrm{A}$, identified inadequacy of informed consent process and documentation as the major deficits among 500 clinical research professionals across India. ${ }^{8}$

Soumil Patwardhan et al. conducted a retrospective analysis of study protocols submitted to the Tata Memorial Hospital IEC and found no difference in the quality and adherence to GCP guidelines between Investigators - initiated trials and sponsored trials with exception of the Informed Consent process. ${ }^{9}$

In a Qualitative analytical study of 162 academic research protocols submitted to our IEC undergraduate $(n=60)$ and postgraduate $(n=102)$ medical students, $91 \%$ of postgraduate medical protocols were approved in comparison to $72 \%$ undergraduate protocols.

Hence, it could be confidently stated that the postgraduate medical student, under the supervision of an experienced research guide can be an apt principal investigator in submitting a research protocol. However, in an enthusiastic quest to finish a 
dissertation/conference presentation/publication, practical translation of the known Research guidelines into Good Clinical Practice are often missed.

\section{CONCLUSION:}

This study has brought out the common deficiencies in the protocols submitted. These deficiencies and steps to correct them are as follows:

Scientific background and rationale of the study hypothesis can be strengthened by a critical literature search from indexed journals that prophesize evidence based medical practice. Technical feasibility can be checked by prior orientation to available infrastructure in the institution of study, forming better collaboration between departments and asking for help from the skilled personnel and conducting a pilot study, if necessary. (with IEC approval)

Being adequately informed, consenting voluntarily to the research procedure and making an independent decision to involve in the research procedures are the

\section{REFERENCES:}

1. Ashley Montagu MF, Primitive Medicine M.D., N. Engl J Med. 1946 July 11; 235:4349DOI: 10.1056/NEJM194607112350203.

2. Richard Eisenberg, Carl Faingold. Knowledge Objectives in Medical Pharmacology. Aspet [Internet]. 2012. Available from:

https://www.aspet.org/uploadedfiles/divisions_and_chapters/as pet_divisions/pharmacology_education/content/educational_as sets/knowledge\%20objectives\%202012\%20edition\%20final.pd f.

3. Peter M Dunn. Perinatal lessons from the past: James Lind (1716-94) of Edinburgh and the treatment of scurvy. Arch Dis Child Fetal Neonatal Ed 1997;76:1 F64-F65 doi:10.1136/fn.76.1.F64

4. Indian Council of Medical Research. Handbook on Ethical guidelines for Biomedical research on Human participants.Oct, 2006.21p. basic rights of the participants. An investigator should keep a transparent, comprehensible and detailed log of the informed consent process to maintain this autonomy of the participant a-priori.

Proper guidance to the ethical guidelines on biomedical research protects the participant's autonomy, provides justice to the population, minimises/ prevents potential harm and promotes beneficence. Updating oneself with evolving regulations together with rationale thinking would serve to remedy the situation. Each institution has templates on the necessary documentation required. Adhering to them would ensure adequacy of documentation

\section{ACKNOWLEDGEMENT:}

We extend our heartfelt gratitude to all the members of the IEC, Government Kilpauk Medical College for guiding us through the conduct of this study.

\section{CONFLICT OF INTEREST: Nil.}

5. University of Aberdeen. Ethics and Research - the theoretical basis [Internet]. Available from: https://www.abdn.ac.uk/clsm/documents/EricM_CERB.pdf.

6. Sukhlecha A. Research publications: Should they be mandatory for promotions of medical teachers? J Pharmacol Pharmacother. 2011;2:221-4. [PMC free article] [PubMed]

7. CIOMS International ethical guidelines for biomedical research involving human subjects. Available from: www.cioms.ch/publications/layout_guide2002.pdf

8. Jadhav M, Bhatt A. Ethics in clinical research in India: A survey of clinical research professionals' perceptions. Perspect Clin Res. 2013 Jan-Mar; 4:4-8.

9. Soumil Patwardhan, Nithya Gogtay, Urmila Thatte, C S pramesh. Quality and completeness of data documentation in an investigator-initiated trial versus an industry-sponsored trial. Indian Journal of Medical Ethics Vol XI No 1 January-March 2014;19-24 